\title{
铁锆氧化物催化剂上苯酚和甲醇气相邻位烷基化反应
}

\author{
徐磊吴淑杰张文祥贾明君刘钢* \\ (吉林大学化学学院, 长春 130021)
}

摘要: 采用共沉淀法制备了不同铅含量的铁铅氧化物催化剂, 考察了它们在苯酚和甲醇气相邻位烷基化反 应中的催化性能. 结果表明, 铁铅两组分氧化物催化剂具有良好的催化活性和邻位选择性, $\mathrm{Zr} 、 \mathrm{Fe}$ 摩尔比为 0.5/100 的催化剂上苯酚的转化率达到 $99.2 \%$, 主要产物邻甲酚和 2,6-二甲酚的选择性分别为 $22.6 \%$ 和 $77.0 \%$, 随着反应温度的提高, 2,6-二甲酚的选择性增加. 铁锆氧化物表面存在的相对较强的酸碱中心可能是获得较 高苯酚转化率和 2,6-二甲酚选择性的主要原因.

关键词：苯酚；甲醇；邻位烷基化；邻甲酚；2,6-二甲酚

中图分类号： $\mathrm{O} 643$

\section{Vapour Phase ortho-Selective Alkylation of Phenol with Methanol over Fe-Zr Oxide Catalysts}

\author{
XU Lei WU Shu-Jie ZHANG Wen-Xiang JIA Ming-Jun LIU Gang * \\ (College of Chemistry, Jilin University, Changchun 130021, P. R. China)
}

\begin{abstract}
Fe- $\mathrm{Zr}$ oxides with different $\mathrm{Zr} / \mathrm{Fe}$ molar ratios were prepared by a co-precipitation method and used as catalysts for the vapor phase ortho-selective alkylation of phenol with methanol. Results showed that the Fe-Zr oxide catalysts had relatively high activity and ortho-selectivity. Among them, the sample with $n_{\mathrm{Z}} / n_{\mathrm{Fe}}=0.5 / 100$ exhibited the highest activity (99.2\% phenol conversion) with a selectivity of $22.6 \%$ to $o$-cresol and $77.0 \%$ to 2,6 -xylenol. An increase in reaction temperature could further improve selectivity for 2,6-xylenol. The presence of relatively strong acid-base sites may be responsible for the high phenol conversion and the high 2,6-xylenol selectivity.
\end{abstract}

Key Words: Phenol; Methanol; ortho-Selective alkylation; o-Cresol; 2,6-Xylenol

苯酚和甲醇气相邻位烷基化反应一直受到广 泛关注, 它的主要产物邻甲酚和 2,6-二甲酚是生产 香料、染料、抗氧化剂、酚醛树脂和聚苯醚树脂等的 中间体, 市场需求量很大 ${ }^{[1,2]}$. 在这个反应中, 目前已 尝试的催化剂体系包括分子篮催化剂、金属氧化物 催化剂和水滑石催化剂等 ${ }^{[3-11]}$, 然而在大多数催化剂 上, 苯酚和甲醇在生成邻位烷基化产物的同时, 也 易生成 O-烷基化产物及其它副产物, 影响产物的 选择性; 另外, 有些催化剂表面易出现积炭和结焦 现象, 导致催化剂活性下降 ${ }^{[12-14]}$.
近期文献报道 ${ }^{[15-21]}$, 一些以铁系元素 $(\mathrm{Fe} 、 \mathrm{Co} 、 \mathrm{Ni})$ 为 主要成分的尖晶石型氧化物催化剂(如 $\mathrm{Zn}_{1-x} \mathrm{Co}_{x} \mathrm{Fe}_{2} \mathrm{O}_{4}$, $\mathrm{Ni}_{1-x} \mathrm{Co}_{x} \mathrm{Fe}_{2} \mathrm{O}_{4}$ 和铬酸钴)对苯酚和甲醇气相邻位烷基 化反应表现出较高的活性, 邻位烷基化的总选择性 (邻甲酚和 2,6-二甲酚选择性之和)可以达到 94\%左 右. 此外, 一种以 $\alpha-\mathrm{Fe}_{2} \mathrm{O}_{3}$ 为主体, $\mathrm{GeO}_{2}$ 或 $\mathrm{SnO}_{2}$ 为助 剂的二元氧化物催化剂也表现出较好的邻位烷基 化选择性, 与工业上普遍采用的 $\mathrm{MgO}$ 体系催化剂相 比, 反应温度可降低 $100 \mathrm{~K}$ 左右 ${ }^{[22]}$. 目前, 有关这些 铁系催化剂上苯酚和甲醇邻位烷基化反应性能及

Received: August 25, 2008; Revised: November 4, 2008; Published on Web: December 16, 2008.

*Corresponding author. Email: lgang@jlu.edu.cn; Tel: +86431-85155390.

教育部博士点基金(20040183003)及国家自然科学基金(20773050)资助项目

C Editorial office of Acta Physico-Chimica Sinica 
催化作用机制等问题的研究已引起人们广泛兴趣.

本文采用共沉淀法制备了一系列不同锆含量的 铁锆氧化物催化剂, 并用于苯酚和甲醇气相邻位烷 基化反应, 考察了 $\mathrm{Zr} / \mathrm{Fe}$ 比、质量空速和反应温度对 催化剂催化性能的影响, 结合 $\mathrm{X}$ 射线衍射 $(X R D) 、 \mathrm{~N}_{2}$ 吸附和程序升温脱附(TPD)等手段, 对催化剂的结 构和表面性质与反应性能之间的关系进行了研究.

\section{1 实验部分}

\section{1 实验试剂}

硝酸铁 $\mathrm{Fe}\left(\mathrm{NO}_{3}\right)_{3} \cdot 9 \mathrm{H}_{2} \mathrm{O}$ (分析纯); 硝酸氧锆 $\mathrm{ZrO}\left(\mathrm{NO}_{3}\right)_{2}$ (分析纯, 辽宁省医药经贸公司试剂厂); 氨 水 $\mathrm{NH}_{3} \cdot \mathrm{H}_{2} \mathrm{O}(25 \%$, 分析纯 $)$.

\section{2 催化剂制备}

不同锆含量的铁锆氧化物催化剂采用共沉淀 法制备. 将一定量的硝酸铁和硝酸氧锆溶于 150 $\mathrm{mL}$ 水中, 搅拌条件下, 采用质量分数为 $10 \%$ 的稀 氨水调节 $\mathrm{pH}$ 值至 9.0, 继续搅拌 $5 \mathrm{~h}$, 经过滤、洗涤 后于 $393 \mathrm{~K}$ 烘箱中干燥 $12 \mathrm{~h}$, 然后在马弗炉中于 $723 \mathrm{~K}$ 下焙烧 $5 \mathrm{~h}$, 制得铁铅氧化物催化剂, 记为 $\mathrm{Fe}-\mathrm{Zr}-\mathrm{O}$. 所制备样品的 $\mathrm{Zr} / \mathrm{Fe}$ 摩尔比分别为 $0.1 /$ $100 、 0.2 / 100 、 0.5 / 100 、 1.0 / 100$ 和 $2.0 / 100$.

\section{3 催化剂的表征}

XRD 分析采用日本 Shimadzu 公司的 Lab 6000 型 $\mathrm{X}$ 射线衍射仪, $\mathrm{Cu} K_{\alpha}$ 靶, 管电压为 $30 \mathrm{kV}$, 管电 流为 $40 \mathrm{~mA} . \mathrm{N}_{2}$ 吸附实验在美国 Micromeritics ASAP 2010 型微孔测量仪上进行, $573 \mathrm{~K}$ 脱气 $12 \mathrm{~h}$, 液氮温度下吸附; 感应偶合等离子体(ICP)测试在美 国 PERKIN ELMER 公司的 Emission Spectrometer

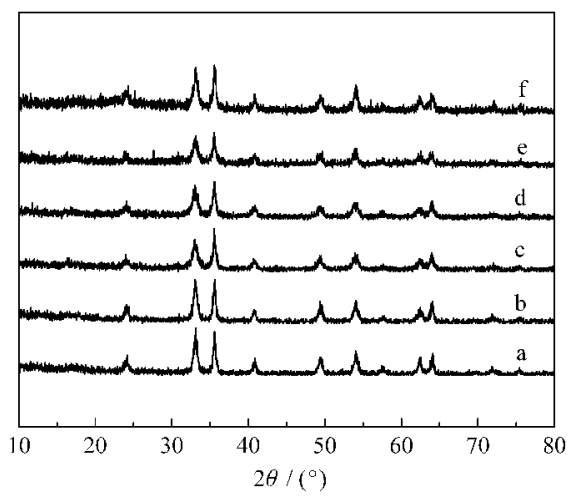

图 $1 \mathrm{Fe}_{2} \mathrm{O}_{3}(\mathrm{a})$ 及不同 $\mathrm{Zr} / \mathrm{Fe}$ 比的 $\mathrm{Fe}-\mathrm{Zr}-\mathrm{O}$ 催化剂( $(\mathrm{b}-\mathrm{f})$ 的 粉末 XRD 图

Fig.1 Powder XRD patterns of catalysts $\mathrm{Fe}_{2} \mathrm{O}_{3}(\mathrm{a})$ and Fe-Zr-O with different $\mathbf{Z r} / \mathbf{F e}$ molar ratios (b-f) $n_{\mathrm{Z}} / n_{\mathrm{Fe}}$ (b) $0.1 / 100$, (c) $0.2 / 100$, (d) $0.5 / 100$, (e) $1.0 / 100$, (f) $2.0 / 100$
上进行; $\mathrm{NH}_{3}-\mathrm{TPD}$ 和 $\mathrm{CO}_{2}-\mathrm{TPD}$ 在自制的装置上测 试, 以氦气作为载气, 热导检测器(TCD)检测; 将 100 $\mathrm{mg}$ 催化剂在氩气气氛中预处理 $1 \mathrm{~h}$, 于 $323 \mathrm{~K}$ 下吸 附 $\mathrm{NH}_{3}$ 气(或 $\mathrm{CO}_{2}$ ) $30 \mathrm{~min}$, 并在该温度下将物理吸 附的 $\mathrm{NH}_{3}$ 气(或 $\mathrm{CO}_{2}$ ) 吹扫干净, 在氦气流中以 $10 \mathrm{~K}$ $\mathrm{min}^{-1}$ 的升温速率进行脱附.

\section{4 催化反应评价}

反应在固定床流动反应器上进行(苯酚与甲醇 的摩尔比 $(n$ (phenol): $n$ (methanol)) 为 $1: 5)$, 催化剂用 量为 $0.8 \mathrm{~g}(40-60$ 目), 在一定的质量空速和反应温 度下进行反应. 反应前在氮气气氛下以 $10 \mathrm{~K} \cdot \mathrm{min}^{-1}$ 的升温速率升至反应温度, 吹扫 $1 \mathrm{~h}$, 反应物通过微 量进样洜由石英玻璃管反应器(直径为 $0.8 \mathrm{~cm}$, 长为 $40 \mathrm{~cm}$ )上端注人, 气化后通过催化剂床层反应. 反应 产物采用日本 Shimadzu 公司的 GC-8A 气相色谱 仪分析, HP-5 毛细管柱, 火焰离子化检测器(FID) 检测, 苯酚的转化率、产物选择性及收率以苯酚为基 准物, 外标并结合归一法进行计算 ${ }^{[14]}$.

\section{2 结果与讨论}

\section{1 铁锆氧化物催化剂的表征}

图 1 为共沉淀法制备的氧化铁和铁锆氧化物 催化剂的 XRD 谱图. 由图 1 可见, 所制备氧化铁样 品具有 $\alpha-\mathrm{Fe}_{2} \mathrm{O}_{3}$ 特征衍射峰 ${ }^{[13]}$. 加人少量锆后, 样品 的特征衍射峰没有发生明显变化, 这可能是由于引 人锆的量相对较低, 没有对氧化铁物种的晶相结构 产生较大影响.

表 1 列出了氮吸附实验测得的比表面、孔容、孔 径和 ICP 测试结果. 由表可见, $\mathrm{Fe}_{2} \mathrm{O}_{3}$ 样品的比表面 积和孔容最小 (分别为 $55 \mathrm{~m}^{2} \cdot \mathrm{g}^{-1}$ 和 $0.18 \mathrm{~cm}^{3} \cdot \mathrm{g}^{-1}$ ); 加 人少量锆 $\left(n_{\mathrm{Z}} / n_{\mathrm{Fe}} \leqslant 1.0 / 100\right)$, 样品的比表面积没有明 显变化, 孔容略有增大; 当锆量增加至 $n_{\mathrm{Zt}} / n_{\mathrm{Fe}}=2.0 /$ 100 时, 样品的比表面和孔容显著提高, 分别为 102 $\mathrm{m}^{2} \cdot \mathrm{g}^{-1}$ 和 $0.31 \mathrm{~cm}^{3} \cdot \mathrm{g}^{-1}$. ICP 结果显示, 焙烧后各样

表 $1 \mathrm{Fe}_{2} \mathrm{O}_{3}$ 及不同 $\mathrm{Zr} / \mathrm{Fe}$ 比 $\mathrm{Fe}-\mathrm{Zr}-\mathrm{O}$ 催化剂的结构参数

Table 1 Selected structural data for $\mathrm{Fe}_{2} \mathrm{O}_{3}$ and Fe-Zr-O catalyst samples

\begin{tabular}{cccccc}
\hline Sample & $n_{\mathrm{ZI}} / n_{\mathrm{Fe}}$ & $n_{\mathrm{ZJ}} / n_{\mathrm{Fe}}{ }^{*}$ & $S_{\mathrm{BET}} /\left(\mathrm{m}^{2} \cdot \mathrm{g}^{-1}\right)$ & $V_{\mathrm{por}} /\left(\mathrm{cm}^{3} \cdot \mathrm{g}^{-1}\right)$ & $D_{\mathrm{por}} / \mathrm{nm}$ \\
\hline 1 & $0.0 / 100$ & - & 55 & 0.18 & 14.3 \\
2 & $0.1 / 100$ & $1.07 \times 10^{-3}$ & 56 & 0.24 & 11.8 \\
3 & $0.2 / 100$ & $1.95 \times 10^{-3}$ & 57 & 0.22 & 12.5 \\
4 & $0.5 / 100$ & $5.30 \times 10^{-3}$ & 57 & 0.23 & 9.0 \\
5 & $1.0 / 100$ & $1.06 \times 10^{-2}$ & 59 & 0.24 & 10.4 \\
6 & $2.0 / 100$ & $2.10 \times 10^{-2}$ & 102 & 0.31 & 8.0 \\
\hline${ }^{*}$ measured by ICP (inductively coupled plasma) chemical analysis
\end{tabular}




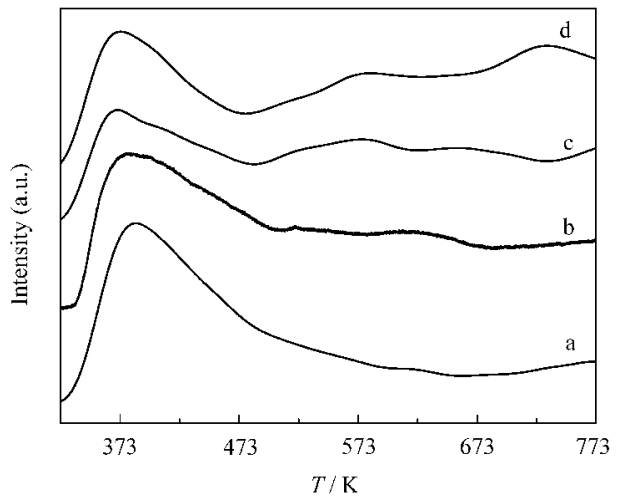

图 $2 \mathrm{Fe}_{2} \mathrm{O}_{3}(\mathrm{a})$ 及不同 $\mathrm{Zr} / \mathrm{Fe}$ 比的 $\mathrm{Fe}-\mathrm{Zr}-\mathrm{O}(\mathrm{b}-\mathrm{d})$ 催化剂 的 $\mathrm{NH}_{3}$-TPD 谱图

Fig.2 $\mathrm{NH}_{3}$-TPD profiles of catalysts $\mathrm{Fe}_{2} \mathrm{O}_{3}(\mathrm{a})$ and

Fe-Zr-O with different $\mathrm{Zr} / \mathrm{Fe}$ molar ratios (b-d) $n_{\mathrm{Z}} / n_{\mathrm{Fe}}:$ (b) $0.2 / 100$, (c) $0.5 / 100$, (d) $2.0 / 100$

品的锆、铁摩尔比与合成时的投料比相近, 表明制备 过程中锆、铁元素没有明显流失.

图 2 为各样品的 $\mathrm{NH}_{3}$-TPD 谱图. 可以看出, $\mathrm{Fe}_{2} \mathrm{O}_{3}$ 样品仅在中心位于 $383 \mathrm{~K}$ 左右有一个 $\mathrm{NH}_{3}$ 的 脱附峰出现, 表明样品表面存在大量的弱酸中心. 加人少量 $\mathrm{Zr}$ 后, 样品除了在 $383 \mathrm{~K}$ 左右出现的脱 附峰外, 还在 563 和 $643 \mathrm{~K}$ 左右出现两个相对较弱 的脱附信号, 说明样品表面同时存在弱酸中心和一 定量的中等强度酸中心; 当 $\mathrm{Zr}$ 量进一步增加, 高温 脱附信号增强且略向高温移动, 表示样品表面中等 强度酸中心的数量增加, 强度略有增大; 当 $\mathrm{Zr}$ 量增 加至 $n_{\mathrm{Z}} / n_{\mathrm{Fe}}=2.0 / 100$ 时, 样品在 $728 \mathrm{~K}$ 附近出现一个 较大的脱附峰, 说明样品表面存在较强的酸中心.

图 3 为各样品的 $\mathrm{CO}_{2}$-TPD 谱图. $\mathrm{Fe}_{2} \mathrm{O}_{3}$ 样品仅 在中心位于 $388 \mathrm{~K}$ 左右有一个 $\mathrm{CO}_{2}$ 的脱附峰出现, 表明样品表面存在大量的弱碱中心. 加人少量 $\mathrm{Zr}$ 后, 样品在 $533 \mathrm{~K}$ 附近出现一个新的脱附信号, 表 明样品表面同时存在一定量中等强度碱中心; 当 $\mathrm{Zr}$ 量进一步增加, 高温脱附信号增强且向高温移

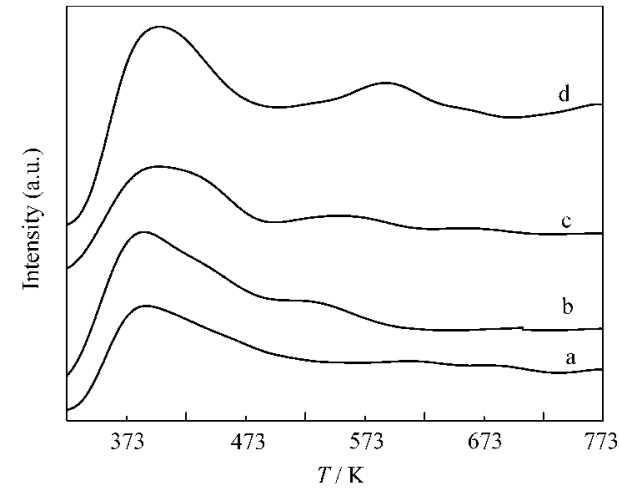

图 $3 \mathrm{Fe}_{2} \mathrm{O}_{3}(\mathrm{a})$ 及不同 $\mathrm{Zr} / \mathrm{Fe}$ 比的 $\mathrm{Fe}-\mathrm{Zr}-\mathrm{O}(\mathrm{b}-\mathrm{d})$ 催化剂 的 $\mathrm{CO}_{2}$-TPD 谱图

Fig.3 $\mathrm{CO}_{2}$-TPD profiles of catalysts $\mathrm{Fe}_{2} \mathrm{O}_{3}$ (a) and

Fe-Zr-O with different $\mathrm{Zr} / \mathrm{Fe}$ molar ratios (b-d)

$n_{\mathrm{Z}} / n_{\mathrm{Fe}}:$ (b) $0.2 / 100$, (c) $0.5 / 100$, (d) $2.0 / 100$

动, 说明样品表面中等强度碱中心的数量增加, 强 度增大; 当 $\mathrm{Zr}$ 量增加至 $n_{\mathrm{Z}} / n_{\mathrm{Fe}}=2.0 / 100$ 时, 高温脱 附信号中心移至 $583 \mathrm{~K}$, 表明样品表面碱中心的强 度进一步增大.

以上结果表明, $\mathrm{Zr}$ 的引入使样品表面出现相 对较强的酸中心和碱中心, 这些酸碱中心的数量和 强度随 $\mathrm{Zr}$ 量的增加而增大.

\section{2 催化剂的性能}

表 2 列出了 $\mathrm{Fe}_{2} \mathrm{O}_{3}$ 和 $\mathrm{Fe}-\mathrm{Zr}-\mathrm{O}$ 氧化物催化剂上 苯酚和甲醇气相邻位烷基化反应结果. 在反应温度 为 $633 \mathrm{~K}$ 条件下, $\mathrm{Fe}_{2} \mathrm{O}_{3}$ 催化剂上苯酚的转化率为 $43.2 \%$, 邻甲酚的选择性为 $84.3 \%, 2,6$-二甲酚选择 性为 $11.1 \%$; 少量 $\mathrm{Zr}$ 加人后 $\left(n_{\mathrm{Z}} / n_{\mathrm{Fe}}=0.1 / 100\right)$, 苯酚的 转化率迅速增加至 $98.2 \%$, 邻甲酚的选择性降低至 $25.3 \%, 2,6$-二甲酚选择性显著提高至 $69.2 \%$; 当 $n_{\mathrm{Z}} / n_{\mathrm{Fe}}=0.5 / 100$ 时, 苯酚的转化率和 $2,6-$ 二甲酚选择 性达到最高, 分别为 $99.2 \%$ 和 $77.0 \%$, 邻位烷基化总 选择性(邻甲酚和 2,6-二甲酚选择性之和)达到 $99.6 \%$ ， 这些指标明显优于文献报道的结果 ${ }^{[15-21]} ; \mathrm{Zr}$ 量进一

表 2 氧化铁和铁锆氧化物催化剂上苯酚和甲醇的烷基化反应性能

Table 2 Catalytic properties of $\mathrm{Fe}_{2} \mathrm{O}_{3}$ and $\mathrm{Fe}-\mathrm{Zr}$-O samples with different $\mathrm{Zr} / \mathrm{Fe}$ ratios ${ }^{\mathrm{a}}$

\begin{tabular}{|c|c|c|c|c|c|c|c|c|}
\hline \multirow{2}{*}{ Sample } & \multirow{2}{*}{$n_{\mathrm{Z}} / n_{\mathrm{Fe}}$} & \multirow{2}{*}{$\begin{array}{c}\text { Phenol } \\
\text { conversion }(\%)\end{array}$} & \multicolumn{4}{|c|}{ Product selectivity (\%) } & \multicolumn{2}{|c|}{ Yield $(\%)$} \\
\hline & & & $o$-cresol & 2,6-xylenol & anisole & others $^{\mathrm{b}}$ & $o$-cresol & 2,6-xylenol \\
\hline 1 & $0.0 / 100$ & 43.2 & 84.3 & 11.1 & 0.1 & 4.5 & 36.4 & 4.8 \\
\hline 2 & $0.1 / 100$ & 98.2 & 25.3 & 69.2 & 0.1 & 5.4 & 24.8 & 67.9 \\
\hline 3 & $0.2 / 100$ & 98.5 & 23.0 & 73.1 & 0.1 & 3.8 & 22.7 & 72.0 \\
\hline 4 & $0.5 / 100$ & 99.2 & 22.6 & 77.0 & 0.1 & 0.3 & 22.4 & 76.4 \\
\hline 5 & $1.0 / 100$ & 98.9 & 30.3 & 65.1 & 0.1 & 4.5 & 30.0 & 64.4 \\
\hline 6 & $2.0 / 100$ & 96.3 & 35.6 & 61.2 & 0.1 & 3.1 & 34.3 & 56.9 \\
\hline
\end{tabular}


表 3 不同反应温度下铁锆氧化物催化剂的反应性能

Table 3 Catalytic properties of Fe-Zr-O sample at different reaction temperatures ${ }^{\mathrm{a}}$

\begin{tabular}{|c|c|c|c|c|c|c|c|}
\hline \multirow{2}{*}{$T / \mathrm{K}$} & \multirow{2}{*}{$\begin{array}{c}\text { Phenol } \\
\text { conversion }(\%)\end{array}$} & \multicolumn{4}{|c|}{ Product selectivity (\%) } & \multicolumn{2}{|c|}{ Yield (\%) } \\
\hline & & $o$-cresol & 2,6-xylenol & anisole & others $^{\mathrm{b}}$ & $o$-cresol & 2,6-xylenol \\
\hline 633 & 99.2 & 22.6 & 77.0 & 0.1 & 0.3 & 22.4 & 76.4 \\
\hline 653 & 99.4 & 17.3 & 77.8 & 0.1 & 4.8 & 17.2 & 77.3 \\
\hline 673 & 99.7 & 15.7 & 80.6 & 0.0 & 3.7 & 15.7 & 80.4 \\
\hline
\end{tabular}

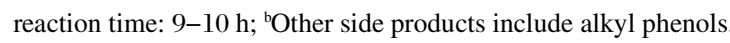

步增加, 苯酚的转化率和 2,6-二甲酚选择性略有降 低. 以上结果说明, 少量锆的引人有利于提高催化 剂活性和 2,6-二甲酚选择性.

表 3 给出了 $\mathrm{Zr} / \mathrm{Fe}$ 摩尔比为 $0.5 / 100$ 的 $\mathrm{Fe}-\mathrm{Zr}-\mathrm{O}$ 样品上反应温度对催化剂性能的影响. 随着反应温 度的升高, 苯酚的转化率仍保持在 $99 \%$ 以上, 邻甲 酚的选择性降低, 2,6-二甲酚的选择性增加; 当反 应温度为 $673 \mathrm{~K}$ 时, 邻甲酚的选择性为 $15.7 \%, 2,6-$ 二甲酚的选择性增加到 $80.6 \%$. 由此可见, 较高的 反应温度更有利于 2,6 -二甲酚的生成.

图 4 给出了质量空速对苯酚和甲醇气相邻位 烷基化反应性能的影响. 随着质量空速的增加, 苯 酚的转化率略有降低, 邻甲酚的选择性明显增加, 而 2,6-二甲酚的选择性降低, 主要副产物苯甲醚的 选择性保持较低水平. 由此可知, 降低质量空速有 利于提高苯酚的转化率和 2,6 -二甲酚的选择性, 降 低邻甲酚的选择性. 根据产物选择性随转化率变化 的趋势可以推测, 2,6-二甲酚主要是由邻甲酚进行

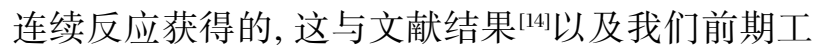
作的结果 ${ }^{[21,23]}$ 相一致. 在反应考察的 $42 \mathrm{~h}$ 内(见图 5),

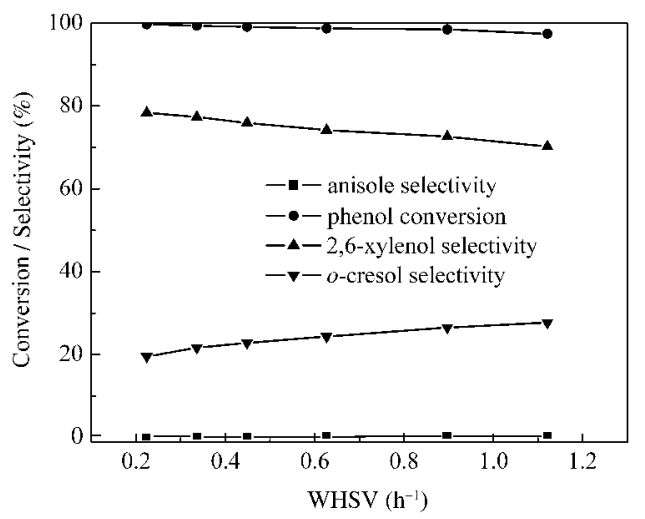

图 4 质量空速对 Fe-Zr-O 催化剂性能的影响

Fig.4 Effect of WHSV on phenol conversion and product selectivity over Fe-Zr-O sample

$n_{\mathrm{Z}} / n_{\mathrm{Fe}}=0.5 / 100$; reaction conditions: $m_{\mathrm{cal}}=0.8 \mathrm{~g} ; n($ phenol $) / n($ methanol $)=$ $1 / 5$; reaction temperature: $633 \mathrm{~K}$; nitrogen flow rate: $3.2 \mathrm{~mL} \cdot \mathrm{min}^{-1}$; reaction time: $9-10 \mathrm{~h}$
苯酚的转化率均保持在 $98 \%$ 以上; 主要产物邻甲酚 和 2,6-二甲酚的总选择性在 $96 \%$ 以上, 显示出较高 的活性和稳定性.

苯酚和甲醇气相邻位烷基化反应属于酸碱催 化反应, 催化剂表面酸碱性质是影响反应性能的重 要因素. 从 $\mathrm{NH}_{3}$-TPD 和 $\mathrm{CO}_{2}$-TPD 结果看, $\mathrm{Fe}_{2} \mathrm{O}_{3}$ 催 化剂上只存在弱酸和弱碱中心, 而 Fe-Zr-O 表面同 时存在一定量的中等强度的酸中心和碱中心. 从反 应结果来看, $\mathrm{Fe}_{2} \mathrm{O}_{3}$ 催化剂上的主要产物是邻甲酚, 2,6-二甲酚的选择性相对较低; 而 Fe-Zr-O 催化剂 上苯酚的转化率和 2,6-二甲酚的选择性显著提高, 邻甲酚的选择性明显降低. 文献[15,16,19]研究表明, 铁系氧化物催化剂(如 $\mathrm{Zn}_{1-x} \mathrm{Co}_{x} \mathrm{Fe}_{2} \mathrm{O}_{4}, \mathrm{Ni}_{1-x} \mathrm{Co}_{x} \mathrm{Fe}_{2} \mathrm{O}_{4}$ 和 $\left.\mathrm{Cu}_{1-x} \mathrm{Co}_{x} \mathrm{Fe}_{2} \mathrm{O}_{4}\right)$ 上, 苯酚主要以立式方式与 $\mathrm{L}$ 酸中心 结合形成酚盐负离子, 甲醇与苯酚邻近的碱性位上 的质子相结合形成碳正离子, 进攻苯酚的邻位. 综 合这些结果, 我们认为弱酸弱碱中心是苯酚邻位烷 基化生成邻甲酚的主要活性中心; 而 $\mathrm{Fe}-\mathrm{Zr}-\mathrm{O}$ 催化 剂上相对较强的酸碱中心更利于苯酚的活化和 2,6-二甲酚的选择性的提高.

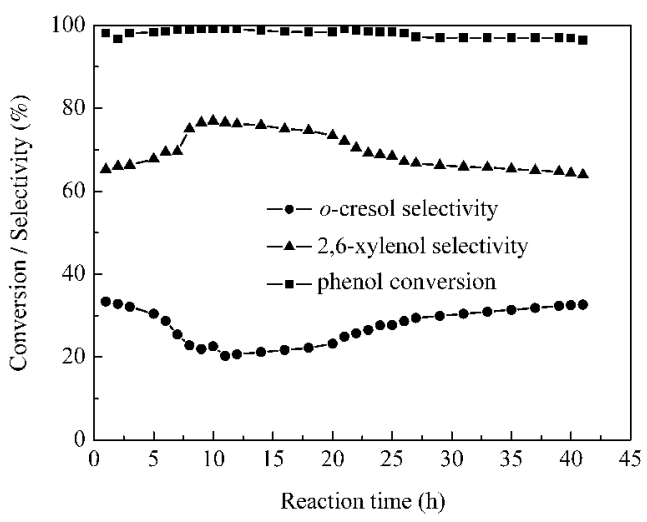

图 5 反应时间对 Fe-Zr-O 催化剂性能的影响

Fig.5 Effect of reaction time on phenol conversion and product selectivity over Fe-Zr-O sample $n_{\mathrm{Z}} / n_{\mathrm{Fe}}=0.5 / 100$; reaction conditions: $m_{\mathrm{cut}}=0.8 \mathrm{~g} ; n(\mathrm{phenol}) / n($ methanol $)=$ $1 / 5$; WHSV $=0.336 \mathrm{~h}^{-1}$; reaction temperature: $633 \mathrm{~K}$; nitrogen flow rate: $3.2 \mathrm{~mL} \cdot \mathrm{min}^{-1}$ 


\section{3 结 论}

采用共沉淀法制备的铁锆氧化物催化剂在苯 酚和甲醇气相邻位烷基化反应中表现出良好的催 化性能, 苯酚的转化率最高可达 $99.2 \%$, 邻位总选 择性在 $95 \%$ 以上, 其中 2,6-二甲酚的选择性较高, 这可能与铁锆氧化物表面存在相对较强的酸碱中 心有关.

\section{References}

1 Wang, Y. L.; Zhang, W. X.; Zhu, X. M.; Zheng, Y. C.; Jia, M. J.; Jiang, D. Z. J. Natural Sci. Heilongjiang. Univ., 2006, 23(2): 150 [王艳力, 张文祥, 朱小梅, 郑艳春, 贾明君, 蒋大振. 黑龙江大学 自然科学学报, 2006, 23(2): 150]

2 Liu, Z. L.; Wang, X. G. Chemical Technology Market, 1999, 10: 10 [刘智凌, 王晓光. 化工科技市场, 1999, 10: 10]

3 Tanabe, K.; Hattori, H.; Sumiyoshi, T.; Tamaru, K.; Kondo, T. J. Catal., 1978, 53: 1

4 Bhattacharyya, K. G.; Talukdar, A. K.; Das, P.; Sivasanker, S. J. Mol. Catal. A, 2003, 197: 255

5 Sato, S.; Koizumi, K.; Nozaki, F. Appl. Catal. A, 1995, 133: L7

6 Sato, S.; Koizumi, K.; Nozaki, F. J. Catal., 1998, 178: 264

7 Gandhe, A. R.; Fernandes, J. B.; Varma, S.; Gupta, N. M. J. Mol. Catal. A, 2005, 238: 63

8 Velu, S.; Swamy, C. S. Appl. Catal. A, 1994, 119: 241

9 Velu, S.; Swamy, C. S. Appl. Catal. A, 1996, 145: 141

10 Choi, W. C.; Kim, J. S.; Lee, T. H.; Woo, S. I. Catal. Today, 2000, 63: 229
11 Jansang, B.; Nanok, T.; Limtrakul, J. J. Phys. Chem. C, 2008, 112 540

12 Santacesaria, E.; Grasso, D.; Gelosa, D. Appl. Catal., 1990, 64: 83

13 Chantal, P. D.; Kaliaguine, S.; Grandmaison, J. L. Appl. Catal., 1985, 18: 133

14 Sad, M. E.; Padro, C. L.; Apesteguia, C. R. Catal. Today, 2008, 133-135: 720

15 Sreekumar, K.; Sugunan, S. Appl. Catal. A, 2002, 230: 245

16 Sreekumar, K.; Sugunan, S. J. Mol. Catal. A, 2002, 185: 259

17 Mathew, T.; Tope, B. B.; Shiju, N. R.; Hegde, S. G.; Rao, B. S.; Gopinath, C. S. Phys. Chem. Chem. Phys., 2002, 4: 4260

18 Mathew, T.; Shylesh, S.; Devassy, B. M.; Vijayaraj, M.; Satyanarayana, C. V. V.; Rao, B. S.; Gopinath, C. S. Appl. Catal. A, 2004, 273: 35

19 Mathew, T.; Vijayaraj, M.; Pai, S.; Tope, B. B.; Hegde, S. G.; Rao, B. S.; Gopinath, C. S. J. Catal., 2004, 227: 175

20 Lazar, K.; Mathew, T.; Koppany, Z.; Megyen, J.; Samuel, V.; Mirajkar, S. P.; Rao, B. S.; Guczi, L. Phys. Chem. Chem. Phys., 2002, 4: 3530

21 Wang, Y.; Zhou, Z.; Jia, M.; Zhu, X.; Zhang, W.; Jiang, D. Catal. Lett., 2005, 104: 67

22 Zhang, H. S.; Xie, G. G.; Qian, M.; Zheng, S. A. J. Fudan Univ. (Natual Science), 1992, 31(4): 456 [张红昇, 谢关根, 钱 敏, 郑绳安. 复旦学报(自然科学版), 1992, 31(4): 456]

23 Wang, Y. L.; Liu, G.; Jia, M. J.; Zhu, X. M.; Zou, X. J.; Zhang, W. X.; Jiang, D. Z. Chem. J. Chin. Univ., 2006, 27(12): 2376 [王艳 力, 刘 钢, 贾明君, 朱小梅, 邹秀晶, 张文祥, 蒋大振. 高等学校 化学学报, 2006, 27(12): 2376] 